\title{
TOWARDS ALGEBRAIC COBORDISM
}

\author{
BY VICTOR SNAITH
}

Communicated by E. H. Brown, Jr., September 9, 1976

\begin{abstract}
A new description of cobordism is given and, by analogy, cobordism theories are defined for an arbitrary ring.
\end{abstract}

1. Let $A$ be a ring with a unit. A cohomology theory, $M A$, might reasonably be called "the algebraic cobordism of $A$ " if

(i) geometry over $A$ gave rise to elements in $\pi_{*}(M A)$, and

(ii) the existence of Chern classes for $A$ induced a transformation of cohomology theories from $M A$ to the algebraic $K$-theory of $A$.

Below I sketch the construction of theories which often satisfy (i) and (ii). Details will appear in [2], [3].

Let $X$ be a homotopy associative and commutative $H$-space. Let $T \subset \pi_{*}^{S}(X)$ be a finite subset of homogeneous elements. To this data is associated a periodic, commutative ring spectrum $X(T), X(T)^{*}$ is the associated cohomology theory. For example, when $X=B U$ and $T$ consists of the generator $B \in \pi_{2}(B U)$, then $X(T)_{2 k}=\Sigma^{2} B U$ and $\epsilon_{2 k}: \Sigma^{2} X(T)_{2 k} \rightarrow X(T)_{2 k+2}$ is equal to

$$
\Sigma^{2}\left(\Sigma^{2} B U\right) \stackrel{h}{\rightarrow} \Sigma^{2}\left(S^{2} \times B U\right) \stackrel{\Sigma^{2}(B \oplus \mathrm{id})}{\longrightarrow} \Sigma^{2}(B U) .
$$

Here $h$ is a Hopf construction and "id" is the identity map of $B U$.

When $X=B G L A^{+}$for a ring $A$ and $T \subset \pi_{*}^{S}\left(B G L A^{+}\right), X(T)^{*}$ is called the algebraic cobordism of $A$ associated with $T$. The terminology is motivated by (a)-(c) of the following result:

THEOREM 1.1. Suppose $\operatorname{dim} Y<\infty$; then:

(a) $B U(T)^{0}(Y) \simeq M U^{2^{*}}(Y)$ if $T=$ (generator of $\pi_{2}(B U)$ );

(b) $B S p(T)^{0}(Y) \simeq M S p^{4 *}(Y)$ if $T=\left\langle\right.$ generator of $\left.\pi_{4}(B S p)\right\rangle$;

(c) $B O(T)^{0}(Y) \simeq M O^{*}(Y)$ if $T=\left\langle\right.$ generator of $\left.\pi_{1}(B O)\right\rangle$;

(d) if $F$ is a finite field and $T$ is a subset of $K_{*}(F)$ then $B G L F^{+}(T)^{0}(Y) \sim$ $=0$;

(e) if $T=\left\langle\right.$ generator of $\left.K_{1}(Z)\right\rangle$ then $B G L Z^{+}(T)^{0}(Y)$ in general is a nontrivial group in which each element is of order 2. 18 F2s.

AMS (MOS) subject classifications (1970). Primary 57A70, 55B15, 55F99; Secondary

Key words and phrases. Cobordism, algebraic corbordism, $K$-theory, algebraic $K$-theory, S-equivalence. 
Theorem 1.1 relates $K$-theory and cobordism very satisfactorily. For example, Adams operations in $K U^{*}$ induce Adams operations in $M U^{*}$ while Adams idempotents in $K U^{*}$ induce Adams idempotents in $M U^{*}$.

The starting point for Theorem 1.1 is the following:

THEOREM 1.2. If $1 \leqslant n \leqslant \infty$ there exist stable equivalences

(i) $B U(n)=\bigvee_{1=k}^{n} M U(k)$,

(ii) $B S p(n)=\bigvee_{1=k}^{n} M S p(k)$,

(iii) $B O(2 n)=\sqrt{ }_{1=k}^{n} B O(2 k) / B O(2 k-2)$ and

(iv) $B S O(2 n+1)=\bigvee_{1=k}^{n} B S O(2 k+1) / B S O(2 k-1)$ when localised away from 2.

2.1. SKetch of Proof of Theorem 1.2. The Becker-Gottlieb transfer is used to embed each classifying space, as a filtered space, into $Q W=\lim _{\longrightarrow} \Omega^{n} \Sigma^{n} W$ for suitable $W$. For example $B U$ is embedded in $Q B U(1)$. The decompositions then follow from the decomposition theorem of [1].

2.2. SKetch of Theorem 1.1. Consider the unitary example. Then

$$
B U(T)^{0}(Y)=\underset{N}{\lim }\left\{\Sigma^{2 N} Y, B U\right\}
$$

where $\{$,$\} means homotopy classes of S$-maps. Hence, by Theorem 1.2, if $\operatorname{dim} Y \leq 4 t$

$$
B U(T)^{0}(Y) \simeq \underset{M}{\lim } \underset{t+M<k}{\bigoplus}\left\{\Sigma^{4 M} Y, M U(k)\right\} \oplus \prod_{t-M \leqslant l} M U^{2 l}(Y) .
$$

A careful study of the $S$-equivalences of Theorem 1.2 and some obstruction theory shows that only the cobordism part of (2.3) remains in the limit.

\section{REFERENCES}

1. V. P. Snaith, Stable decomposition of $\Omega^{n} S^{n} X$, J. London Math. Soc. (7) 2 (1974), $577-583$.

2. Cobordism and the stable homotopy of classifying spaces, Quart. J. Math. Oxford Ser. (to appear).

3. - Algebraic cobordism and $K$-theory (to appear).

DEPARTMENT OF MATHEMATICS, UNIVERSITY OF WESTERN ONTARIO, LONDON, ONTARIO, CANADA 